\title{
Research on Ensemble Learning-based Housing Price Prediction Model
}

\author{
Bowen Yang, Buyang Cao \\ Tongji Univeristy, School of Software Engineering, Shanghai, China \\ bowenyang@yeah.net \\ Caobuyang@tongji.edu.cn
}

Keywords: Housing price, Ensemble Learning, Random forest, Gradient Boosting Decision Tree, XGBoost, Housing Price Prediction Model

\begin{abstract}
Housing price is influenced by multiple factors. The existing housing price forecasting model usually belongs to the so called single predictor model, whose prediction accuracy is not ideal and the over-fitting phenomenon often happens due to the data noise. To resolve these issues, this paper proposes an ensembe lerning-based housing price prediction model incorporating various predictors. To evaluate the effectiveness of the proposed model, extra trees, random forest, GBDT and XGB algorithms are selected for the benchmarks. The dataset used is the California housing price available over the web. The results demonstrate that the proposed method can improve the predicting accuracy and stability compared with other four single prediction models.
\end{abstract}

\section{Introduction}

Real estate is not only a key sector in the national economy, but also one of the people's major concerns. Due to the housing demands, people's attention to the housing price continues to increase. Therefore, it is critical to provide accurate predictions of housing prices. Housing price is impacted by multiple factors ([2], [10]) including time and space, house ages, surrounding conditions, communities, transportation, etc. Existing prediction models are usually single predictor ones, i.e., a single forecasting model is applied to the prediction. The prediction accuracy of this model is not satisfactory when datasets are noisy [4]. Some simple ensemble models such as random forest would encounter over-fitting phenomenon when the data contain more noise. To address these issues, the paper proposes an ensemble learning ([1], [11]) based housing price prediction model. The model is built upon multiple single predictors (they will be called base predictors in the following discussions) including random forest (RF), extra trees (ET), GBDT, and XGB.

Random forest [7], whose basic unit is a decision tree, is an ensemble algorithm $/ \mathrm{model}$ employing multiple trees. It shows its superiority in many application areas. It is capable of handling high dimensional data without feature selection. It can get an unbiased estimation of the internal generation error during the forest generating process, and the generalization capability is good. Nevertheless, random forest may suffer overfitting in some classification or regression problems where noise occurs.

Extra trees, also known as Extremely Randomized Trees, is the combination of decision trees. 
Similar to the random forest, it randomly selects partial features to construct a tree. Extra trees directly use training samples to construct random trees and modify the way of bagging. Therefore, when data are noisier or the dataset is large, this methodology performs better than the standard random forest. However, due to more randomly sampled data, some selections are not satisfactory and the quality of prediction results fluctuates greatly.

GBDT (Gradient Boosting Decision Tree) [8] is an iterative decision tree algorithm. This algorithm consists of multiple decision trees whose conclusions form the final answer, and GBDT is considered to have a strong generalization capability. The core of a GBDT is composed of regression trees. Therefore, most GBDTs are used for regression predictions. Although a GDBT does not need to perform complex feature engineering and transformation, it is not quite suitable for the problems with high-dimensional features.

XGBoost (XGBT) [6] is an open-source software library including the gradient boosting framework aiming at providing a "scalable, portable and distributed gradient boosting library". Other than running on a single machine, it also supports the distributed frameworks. Using XGBoost, models can be traibed more efficiently and better prediction results can be obtained.

Based on the above discussions, the existing methods/predictors cannot provide satisfactory and stable results if they are applied individually. They are impacted negatively by the noise in datasets. Hence, the paper will develop an ensemble learning based on prediction model by incorporating the above mentioned four predictors to obtain better prediction outcomes. The procedure of creating the proposed model or method may be described briefly as follows.

The stacking method ([5], [9], [11]) of ensemble learning is applied to construct the proposed model. It first partitions the data sets (see the details in the following sections), and then uses each base predictor to conduct the predictions based on the extract features related to housing prices. Specifically, the first part of the dataset is used for training, and the second part is employed for testing these base predictors. At the end, taking the testing results as the inputs, the high-level (ensemble) model is finally trained as the prediction model.

In the next section it is going to present a model, the training process, and some computational experiments. Then, the paper concludes with remarks.

\section{Ensemble learning based prediction model}

The following picture (figure 1) depicts the overall model training and ensembling process for the proposed model, where the dataset refers to the California housing price data.

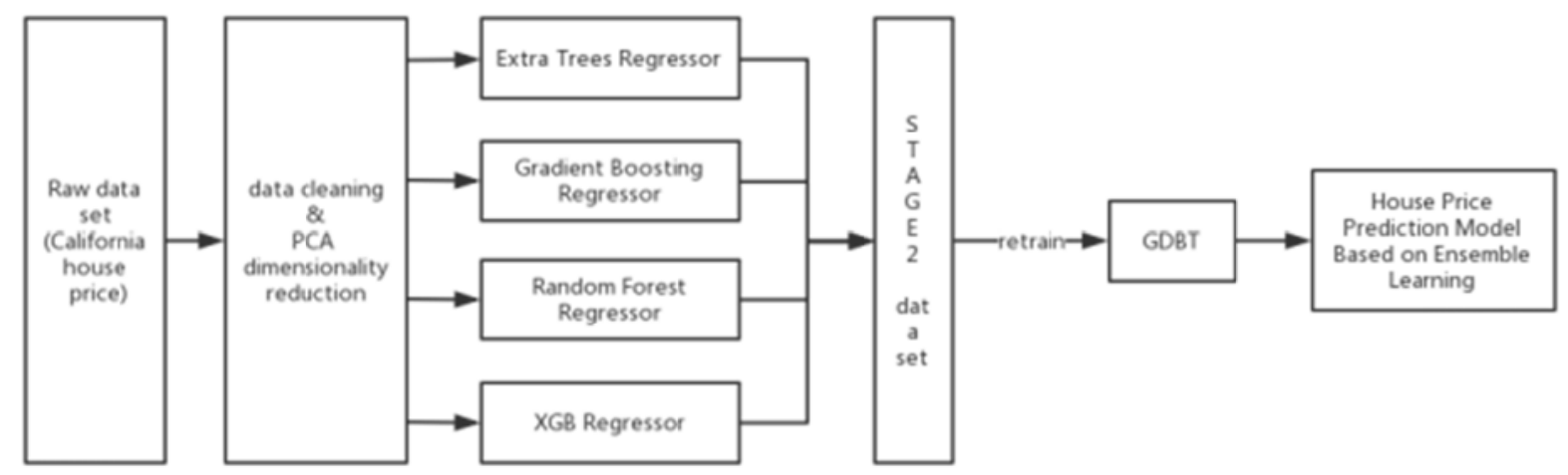

Figure 1. Ensemble training process of the proposed model 


\subsection{Data processing}

This paper uses California housing price data for training and evaluating the model. According to the analysis of the original dataset, it contains numeric and non-numeric features and some records miss data contents. The dataset needs to be cleaned before being used for training and testing. The paper analyzed the original dataset and found that the feature 'ocean_proximity' is expressed with String format, it is not a friendly way for machine learning, so the ocean_proximity feature data would be transformed to numeric data. The ocean_proximity data contain data of five types such as NEAR BAY, INLAND, NEAR OCEAN.etc. So the paper adds five features depending on these five data types into the original dataset and marks the corresponding data as $\mathbf{1}$, the others as 0 , for example, if the house is near bar, the Ocean_proximity_NEAR_BAR is marked as 1, the other four features as 0 , the details can be seen from Table 1 and Table 2. Finally, the non-numeric data of the selected features are transformed to numeric ones and the missing data will be replaced properly. The samples of both original and processed data are shown in the follows tables respectively:

Table 1. Original data sample

\begin{tabular}{|c|c|c|c|c|c|c|c|c|}
\hline longitude & latitude & houving median_age: & rooms & edrooms & & households & median_income & median_house_value ocean_proximity \\
\hline-122.23 & 37.83 & 41 & 880 & 129 & 322 & 126 & 8.3252 & 452600 NEAR BAY \\
\hline-1222 & 37.86 & 21 & 7099 & 1106 & 2401 & 1138 & 8.3014 & 358500 NEAR BAY \\
\hline-122.20 & 37.85 & 52 & 1967 & 190 & 496 & $17 n$ & 7.2574 & 352100 NEAR BAY \\
\hline-122.25 & 37.85 & 52 & 1274 & 235 & 558 & 219 & 5.6431 & 341300 NEAR BAY \\
\hline-122.25 & 37.85 & 52 & 1627 & 280 & 565 & 259 & 3.8462 & 342200 NEAR BAY \\
\hline-122.25 & 37.85 & 52 & 919 & 213 & 413 & 193 & 4.0368 & 269700 NEAR BAY \\
\hline-122.25 & 37.84 & $\mathbf{5 2}$ & 2535 & 489 & 1094 & $\mathbf{5 1 4}$ & 3.6591 & 299200 NEAR BAY \\
\hline .122 .25 & 37.84 & 52 & 3104 & 687 & 1157 & 647 & 3.12 & 241400 NEAR BAY \\
\hline-122.26 & 37.84 & 42 & 2555 & 665 & 1206 & 595 & 2.0804 & 226700 NEAR BAY \\
\hline
\end{tabular}

Table 2. Processed data sample

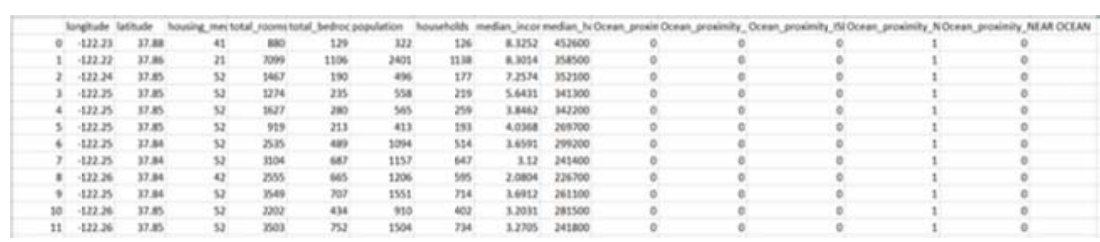

Each record of the dataset contains 15 attributes, and the dataset consists of 20,000 records. A sample matrix $(20,000 \times 15) X=\left[x_{1}, x_{2}, \ldots, x_{n}\right]^{T}$ is constructed based on the number of records and features where $\mathrm{n}$ is the number of records or samples. However, some features do not necessarily contribute to the variable, i.e., the housing price, to be predicted and they even act as noise. Therefore, the principal component analysis (PCA) method is applied to reduce the dimension for better results. Particularly, The Karhunen-Loeve Transform (KLT) [12] is applied to perform the PCA task. A new sample matrix or data collection $L$ will be generated after the PCA process. In the following training and testing procedures the paper would use the dataset $L$ after the dimensional reduction by PCA.

\subsection{Training base predictors}

As mentioned earlier, the ensemble learning based prediction model proposed in this paper is created based on the stacking ensemble learning method ([5], [11]), where ET, RF, GBDT, and XGB are base predictors. In this case, it is necessary to train these selected base predictors. For each base predictor, it applies the associated regression model and uses the new sample data $L$ after the dimensional reduction described above as the input training data. As usual, the dataset is divided into two parts, whereas $99.5 \%$ of it is for training and $0.5 \%$ of it is for testing. During the testing process, the model parameters are adjusted to achieve the more satisfactory results of the underlying models. The parameters to be set for the individual base predictors are listed as follows:

max_features: max features allowed to use in each predictor; 
n_estimators: the number of trees of each predictor;

colsample_bytree: specifying the number of columns per random sample max_depth: the max depth of a node in one tree of a predictor subsample: the ratio of the input data to be sampled.

Table 3. Model parameters after training

\begin{tabular}{|c|c|c|c|c|}
\hline ET & max_features $=6$ & n_estimators $=100$ & & \\
\hline RF & max_features=6 & n_estimators $=100$ & & \\
\hline GBDT & max_features $=12$ & n_estimators $=500$ & max_depth=8 & sulbsample $=0.8$ \\
\hline XGB & max_features $=12$ & $\begin{array}{r}\text { n_estimators }=200 \\
\text { Colsample } \\
\text { _bytree }=0.8\end{array}$ & max_depth=8 & sulbsample $=0.8$ \\
\hline
\end{tabular}

After having tuned the parameters, the resultant models/predictors can be used for conducting predictions. The figure 2 presents the prediction results of all four base predictors and the (sampled) real housing prices for comparisons.
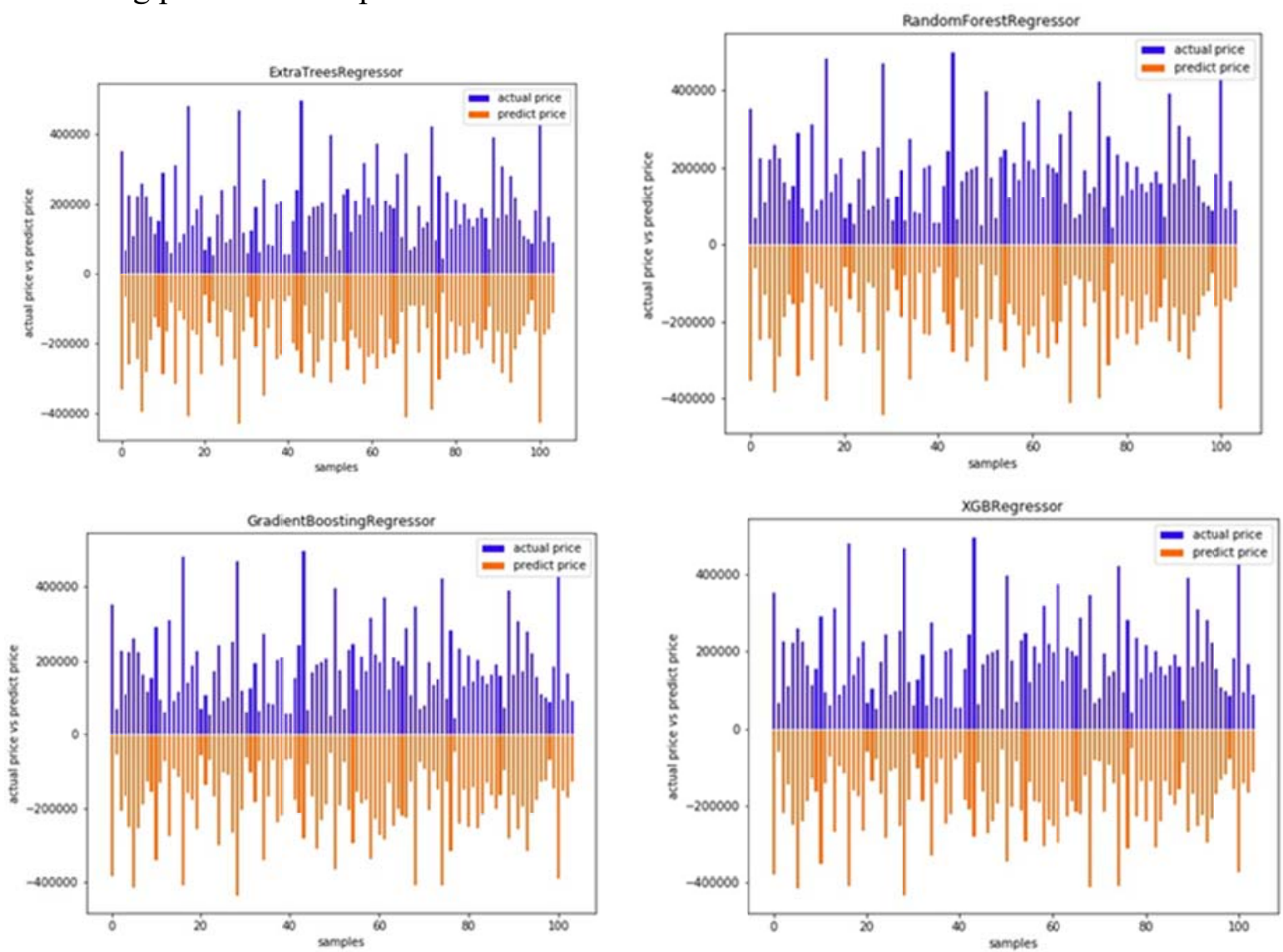

Figure 2. The results obtained by four basic predictors 
The following table (table 4) lists the loss function values measured by mean square errors, which can be applied to evaluate the performance of each predictor.

Table 4. Mean square errors of four base models

\begin{tabular}{|c|c|}
\hline Predict model & Mean Squared Error \\
\hline Extra Trees & 44216.900081 \\
\hline Random Forest & 44625.093537 \\
\hline Gradient Boosting & 43764.930335 \\
\hline XGB & 43279.231065 \\
\hline
\end{tabular}

Based on the outcomes, it is not difficult to find out that both GBDT and XGB produce better prediction results than ET and RF do in terms of MSE (mean sqaure error). The next section will present more details on how to apply these four base predictors to construct an ensemble model. Further computational experiments and benchmarks are carried out to evaluate the effectiveness/performance of the resulted ensemble model.

\subsection{Model ensemble and training}

In the previous section, four base predictors are trained and the corresponding forecasting models and results are obtained. These four base predictors will be employed to create the final ensemble model. The entire training process for the ensemble model is performed in the following two stages:

- Assuming that the given sample dataset $L=\left\{\left\{x_{i}, y_{i}\right\}, i=1,2, \ldots, n\right\}$ contains $n$ tuples (samples), where $x_{i}$ is the feature vector of the $i$-th sample after the dimensional reduction or PCA, $y_{i}$ is the $i$-th target or real value. Specifically, in this case, there are 20,000 samples with each having a certain number of features and $y_{i}$ is the true housing price associated with ith sample.

In order to prevent the over-fitting situation from happening, the principle of crossvalidation is applied to construct the second-level dataset. As Stacking ensemble learning method is used to predict the house price data, the four basic predictors are needed to predict once to get the prediction result, then the result and a part of original dataset got before are merged as the second-level dataset using a well-perform predictor to predict again, so as to avoid some of the predictors' decision, and ensemble the four predictors' predicting result as the final predicting result. The original dataset $L$ is randomly divided into $k$ parts (they are called subsamples in the following discussion) $L_{1}, L_{2}, \ldots, L_{k}$. Furthermore, define $L_{i}$ and $L_{i}=L-L_{i}$, for $i=1,2, \ldots, k$ are defined to be the $i$-th cross-validation training and testing datasets respectively.

Four base predictors will be trained separately using the training datasets and four resultant base predictors are obtained. The prediction result achieved by the $j$-th predictor on $i$-th sample in the testing dataset is denoted by $Z_{i j}$. Since the number of subsamples is k, thus, the training process repeats $k$ times, whereas each subsample will be used for performing $t$ predictions and obtaining the corresponding predicting results. These predictions together with the target values of the corresponding samples form the dataset used for the second-stage, namely, $L_{c v}=\left\{\left(Z_{i 1}\right.\right.$, $\left.\left.Z_{i 2}, \ldots, Z_{i t}, y_{i}\right), i=1,2, \ldots, n\right\}$. Through this process, the training dataset of the ensemble training is a new dataset consisting of all prediction results and the corresponding target values (housing prices in this case). At the end, the final ensemble prediction model is obtained upon $L_{c v}$. 
- According to the results presented in the previous section, it is found that GDBT model is a better choice for the ensemble model because it possesses relatively low MSE. Hence GDBT is utilized as the training model again for the final ensemble model. Similarly, the parameters such as n_estimators, learning_rate, subsample, etc. listed in table 3 are adjusted based on the best solution obtained during this training process. The computational experiements reveal that the setting of $n \_$estimators $=100$, subsample $=0.75$ can achieve the best solution. At the end, the ensemble learning based housing price prediction model is trained and constructed. The effectivness of this model will be evaluated through a series of computational experiements introduced in the next section.

\subsection{Results analysis}

Similar to the evaluating processes for four base predictors mentioned above, the paper compares the predicted housing prices from the ensemble model to the actul ones to verify the accuracy or performance of the ensemble model. Figure 3 depicts the predicted houing prices against the actual ones.

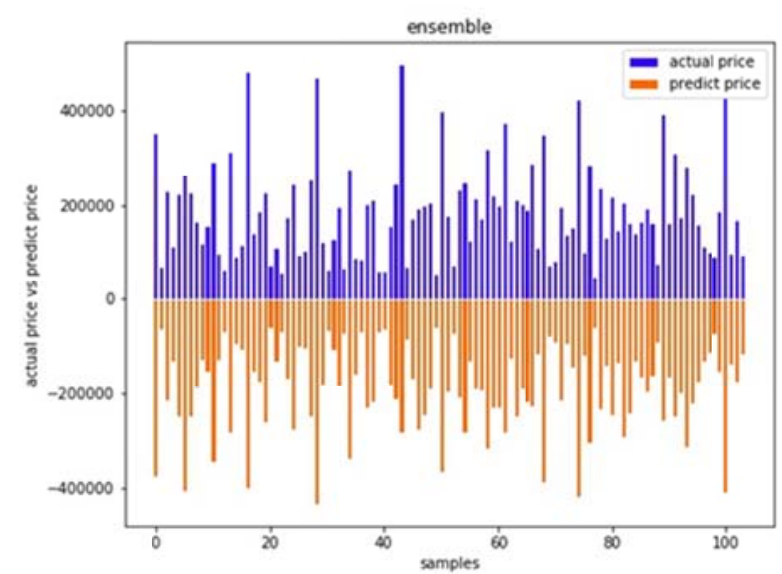

Figure 3. Prediction results obtained by the ensemble model

The horizontal axisin represents the data samples, and the vertical axis shows the housing prices where the blue is for the actual prices while the orange is for the predicted ones. It is hard to validate the accuracy and effectiveness of the ensemble model visually, again the loss function (mean square error) is used to evaluate the predicting accuracy (effectiveness). The complete results are shown in table 5. For comparison purpose, the MSE (mean of square error) of four base predictors is also listed in the table. 
Table 5. MSE for all models

\begin{tabular}{|l|l|}
\hline Predict model & Mean Squared Error \\
\hline Extra Trees & 44216.900081 \\
\hline Random Forest & 44625.093537 \\
\hline Gradient Boosting & 43764.930335 \\
\hline XGB & 43279.231065 \\
\hline Ensemble Model & 41811.422310 \\
\hline
\end{tabular}

It is not difficult to recognize the prediction results obtained by the ensemble model with the lowest MSE, which is reduced by $6.7 \%$ on average. The computation results indicate that the ensemble model is able to provide the most accurate predictions in general.

A base predictor would have its pros and cons, and it might not be able to work on all datasets with the universal superiority. By applying the ensemble techniques, the advantages of the underlying base predictors or models are strengthened while the shortcomings of these base models are avoided. The ensemble model demonstrates its effectiveness in dealing with datasets with noise and overfitting problems.

\section{Conclusion}

This paper presents a housing price prediction model built upon ET, RF, GBDT and XGB by applying the stacking ensemble learning methodology. The process of building an ensemble model includes extracting relevant features from California housing price data, performing the dimensional reduction, and training the model respectively. During the ensemble model construction, the individual prediction results are used as the inputs for training the ensemble predictor, which leads to the final prediction model. The advantage of this model is that it can improve the prediction accuracy and effectively avoid the overfitting when the dataset contains noise or too many features. At the same time, the ensemble model is able to produce more stable results. Although the proposed ensemble model functions cannot be claimed better than each base predictor consistently for all scenarios, the outcomes obtained by the ensemble model are very promising. It also encourages people to apply the similar technology to other machine learning problems in the future.

\section{Acknowledgments}

This work was partially supported by the China Intelligent Urbanization Co-Creation Center [grant number CIUC20150011].

\section{References}

[1] Oza N C. Online Ensemble Learning. The AAAI conference on artificial intelligence, 2000.

[2] Park B. and Bae J K. Using machine learning algorithms for housing price prediction. Expert Systems with Applications, 42: 2928 - 2934, 2015.

[3] Bourassa S C, Cantoni E, and Hoesli M. Predicting House Prices with Spatial Dependence: A Comparison of Alternative Methods. Journal of Real Estate Research, 32: 139-160, 2010.

[4] Rajan U, Seru A, and Vig V. The failure of models that predict failure: Distance, incentives, and defaults. Journal of Financial Economics, 115: 237 - 260, 2015.

[5] Burger E M and Moura S J. Building Electricity Load Forecasting via Stacking Ensemble Learning Method with 
Moving Horizon Optimization. 2015.

[6] Rodriguez J J, Kuncheva L I, and Alonso C J. Rotation Forest: A New Classifier Ensemble Method. IEEE Transactions on Pattern Analysis and Machine Intelligence, 28: 1619 - 1630, 2006.

[7] Liu J and Wu C. A gradient-boosting decision-tree approach for firm failure prediction: an empirical model evaluation of Chinese listed companies. Journal of Risk Model Validation, 11: 43 - 64, 2017.

[8] Sikora R. A modified stacking ensemble machine learning algorithm using genetic algorithms. Handbook of Research on Organizational Transformations through Big Data Analytics. IGI Global, 43 - 53, 2015.

[9] James Brown and John Smith. How not to cite papers. In John Smith, editor, Proceedings of the First International Conference on Modern Bibliometrics (MODBIB 2009), pages 20-30, Pasadena (CA), USA, July 25-28 2009.

[10] Glaeser E L and Nathanson C G. An extrapolative model of house price dynamics. Journal of Financial Economics, 2017.

[11] Zhou Z H. Ensemble learning. Encyclopedia of biometrics, 2015: 411-416.

[12] https://en.wikipedia.org/wiki/Karhunen\%E2\%80\%93Lo\%C3\%A8ve_theorem 